01

\title{
Возможность управления динамикой и структурой магнитного солитона в трехслойной ферромагнитной структуре
}

\author{
(C) Е.Г. Екомасов ${ }^{1,2}$, В.Н. Назаров ${ }^{3,4}$, К.Ю. Самсонов ${ }^{1}$, Р.Р. Муртазин ${ }^{2}$ \\ ${ }^{1}$ Тюменский государственный университет, Тюмень, Россия \\ ${ }^{2}$ Башкирский государственный университет, Уфа, Россия \\ ${ }^{3}$ Институт физики молекул и кристаллов - обособленное структурное подразделение \\ Уфимского фредерального исследовательского центра РАН, Уфра,Россия \\ ${ }^{4}$ Башкирский государственный медицинский университет, Уфа, Россия \\ E-mail: EkomasovEG@gmail.com
}

Поступило в Редакцию 25 января 2021 г.

В окончательной редакции 15 февраля 2021 г.

Принято к публикации 17 февраля 2021 г.

Рассмотрены генерация и возбуждение магнитного солитона в трехслойном ферромагнетике постоянными магнитными полями и полями переменной частоты и малой амплитуды при наличии диссипации в системе. Анализ решений уравнения движения в переменном поле показывает возможность увеличения со временем при определенных условиях амплитуды магнитного солитона. На резонансный эффект влияют также геометрические параметры тонкого слоя: при большой ширине слоя возбуждается еще и трансляционная мода колебаний солитона.

Ключевые слова: трехслойный ферромагнетик, магнитный солитон, локализованные магнитные неоднородности, авторезонанс.

DOI: 10.21883/PJTF.2021.10.50966.18718

В последнее время появилось много работ по использованию магнитных неоднородностей типа магнитных вихрей и скирмионов в спинтронных устройствах $[1,2]$. Конкуренцию им могут создать магнитные неоднородности солитонного типа, имеющие много схожих динамических свойств [3]. Появление новых экспериментальных методик, позволяющих изучать процессы формирования и распространения локализованных волн намагниченности нанометровых размеров и взаимодействия их с доменными границами (ДГ) [4-6], также стимулировало возросший прикладной интерес к данной теме [7]. Ключевым вопросом для создания новых устройств является нахождение условий для генерации устойчивых локализованных волн намагниченности типа магнитных солитонов и бризеров. Известно, что в области магнитного „дефекта“, являющегося 1D-, 2Dили 3D-потенциальной ямой для магнитной неоднородности (см., например, [8-11]), это вполне возможно. В одномерном случае одним из возможных путей при создании таких магнитных „дефектов“ является создание многослойных магнитных структур, представляющих собой периодически чередующиеся слои двух материалов с различными физическими свойствами, например магнитной анизотропией [12]. Одним из возможных способов управления динамикой намагниченности являются приложение внешнего магнитного поля [13] и учет затухания в системе. В настоящей работе рассматривается возможность управления параметрами магнитного солитона в трехслойном ферромагнетике при помощи постоянных магнитных полей и полей переменной частоты и малой амплитуды с использованием авторезонансной модели управления при наличии диссипации в системе.

Рассмотрим трехслойную ферромагнитную структуру, состоящую из двух широких одинаковых слоев, разделенных тонким слоем с измененными значениями параметра магнитной анизотропии [14]. Параметры анизотропии будем считать функциями координаты $x$, направленной перпендикулярно границе раздела слоев. Обычно при решении динамических задач удобно перейти к сферическим координатам $\theta$ и $\varphi$ вектора намагниченности $\mathbf{M}$, где $0 \leqslant \theta \leqslant 2 \pi-$ угол в плоскости $y z$ между направлением вектора М и осью легкого намагничивания (ось $O z$ ), $-\pi / 2<\varphi<\pi / 2-$ угол, описывающий выход М из плоскости ДГ. Учитывая в плотности энергии магнетика обменное взаимодействие и анизотропию и считая $\varphi \ll 1$, уравнение движения для намагниченности можно представить в виде

$$
\Delta \theta-\ddot{\theta}-\frac{1}{2} f(\mathbf{r}) \sin 2 \theta=h \sin \theta+\alpha \dot{\theta},
$$

где $f(\mathbf{r})=K_{1}(x) / K_{1}^{0}-$ функция, определяющая пространственную модуляцию константы анизотропии, $K_{1}^{0}$ - константа анизотропии в толстых слоях, $h=\left(H_{Z} / 4 \pi M_{S}\right) Q^{-1} \quad$ и $\quad \alpha=\alpha_{0} / \sqrt{Q} \quad$ - нормированные внешнее магнитное поле и константа затухания, $Q=K_{1}^{0} /\left(2 \pi M_{S}^{2}\right)$ - фактор качества материала, $\alpha_{0}$ - константа затухания, время $t$ нормировано на $4 \pi M_{S} \gamma \sqrt{Q}$, координата $x$ нормирована на ширину статической блоховской ДГ. Функцию $f(x)$ в одномерном случае для простоты будем моделировать в форме 
прямоугольника:

$$
f(x)= \begin{cases}1, & |x|>W_{x} / 2 \\ K, & |x|<W_{x} / 2\end{cases}
$$

где $W$ - параметр, характеризующий ширину тонкого слоя, $K$ - величина, нормированная на константы магнитной анизотропии в области тонкого слоя. Следует заметить, что другие виды функции (2), например гауссова типа, ведут к изменению набора параметров, приводящих к образованию магнитного солитона и его собственной частоты колебаний [9].

Уравнение (1) решалось численно с использованием явной схемы интегрирования [14]. Для этого выбиралась трехслойная схема решения с аппроксимацией производных на пятиточечном шаблоне типа „крест“ [11]. Схема численного эксперимента выглядит следующим образом. Распределение намагниченности в начальный момент времени задавалось в виде блоховской ДГ $\theta_{0}(x)=2 \operatorname{arctg}\left(e^{x}\right)$, находящейся далеко от тонкого слоя. Известно, что при определенных значениях параметров тонкого слоя при прохождении ДГ с постоянной скоростью через него образуется магнитная неоднородность в виде магнитного бризера или солитона. Случай магнитного бризера в переменном внешнем магнитном поле был нами рассмотрен ранее в [14]. Рассмотрим теперь магнитные неоднородности солитонного типа. Скорость движения блоховской ДГ возьмем на большом расстоянии от тонкого слоя равной 0.85 в безразмерных единицах, константу затухания - равной 0.001. Начиная с определенных величин параметров $W=1.9, K=-1.4$ в области тонкого слоя наблюдаем образование магнитной неоднородности солитонного типа. При $W \geqslant 2, K<-1.8$ наблюдаем образование магнитного антисолитона с противоположным по отношению к солитону направлением намагниченности в его центре.

Если приложить постоянное внешнее магнитное поле против направления намагниченности в центре магнитного солитона (МС), то, очевидно, как и для случая магнитных вихрей в спин-вентильных структурах [11], можно ожидать переключения направления намагниченности в центре МС при некоторой критической величине магнитного поля. При $h=0.6, W=2, K=-2$ наблюдалось подобное переключение и превращение магнитного солитона в антисолитон (рис. 1). Следует отметить, что при этом, как и для случая магнитных вихрей в спин-вентильных структурах [11], необходимо прикладывать достаточно большую величину постоянного поля.

Рассмотрим далее случай применения переменного внешнего магнитного поля и используем явление авторезонанса для управления динамическими характеристиками магнитного солитона. Известно, что применение авторезонансных моделей управления позволяет существенно уменьшить величину внешнего воздей-

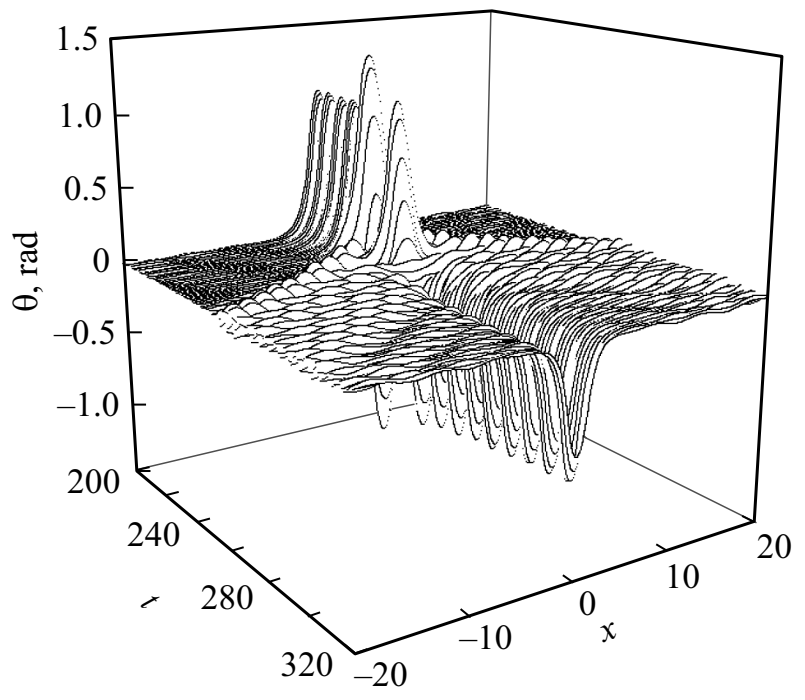

Рис. 1. Перемагничивание магнитной неоднородности типа солитон: до момента времени $t=220$ при $h=0.32$ существовал покоящийся солитон, после $t=220$ при включении поля величиной $h=0.6$ происходило переключение. $W=2$, $K=-2$, координаты центра области дефекта $x=0$.

ствия на систему [15-17]. Поле имеет вид, где частота $\omega=\omega_{0}+\mu t\left(\omega_{0}-\right.$ собственная частота солитона, $\mu$ - малый параметр). Рассмотрим случай $K=-1.4$ и $W=2$, когда в отсутствие поля после прохождения ДГ через тонкий слой в нем генерируется МС, амплитуда которого испытывает колебания (рис. $2, a)$. При $h_{0}=0.1$, $\mu=0.01$ и $\omega_{0}=0.83$ при прохождении ДГ через тонкий слой будем наблюдать генерацию уже магнитного антисолитона. Если в отсутствие магнитного поля амплитуда солитона затухает со временем, то в переменном поле определенной частоты (связанной с собственной частотой колебаний магнитного солитона) амплитуда магнитного антисолитона возрастает в 2 раза (рис. 2), но дальнейший рост амплитуды ограничивается за счет излучения спиновых волн. При увеличении параметра $K$ в переменном магнитном поле будет наблюдаться аналогичная ситуация: амплитуда колебаний магнитного антисолитона возрастает в 2 раза, но только с другой измененной частотой, так как частота антисолитона зависит от параметров тонкого слоя. Такое ограничение на увеличение амплитуды колебаний вызвано тем, что центр антисолитона уже не остается в центре тонкого слоя, а возбуждается еще и трансляционная мода его колебаний вдоль координаты $x$, сопровождающаяся излучением объемных спиновых волн. Наиболее отчетливо эти волны проявляются в отсутствие затухания (рис. 3). Случай достаточно малой ширины тонкого слоя приводит к исчезновению трансляционной моды колебаний магнитного солитона. Здесь можно добиться большего увеличения амплитуды солитона (практически на порядок) в переменном магнитном поле по сравнению со случаем без поля. 

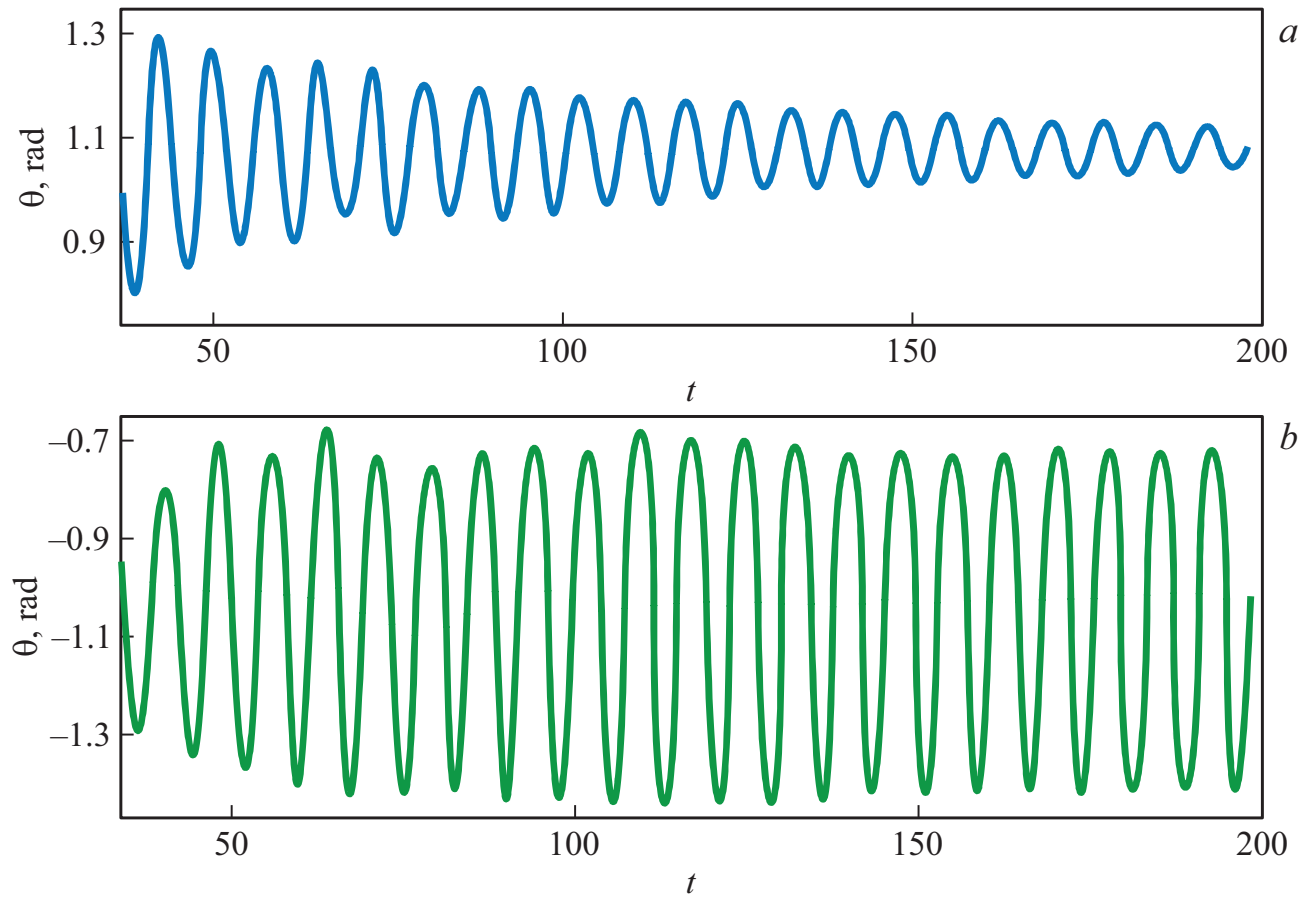

Рис. 2. Зависимость амплитуды солитона от времени без поля $(a)$ и в переменном поле $(b), h_{0}=0.1$, параметр $\mu=0.01$, начальная частота поля 0.83 , параметры ямы $W=2, K=-1.4$.
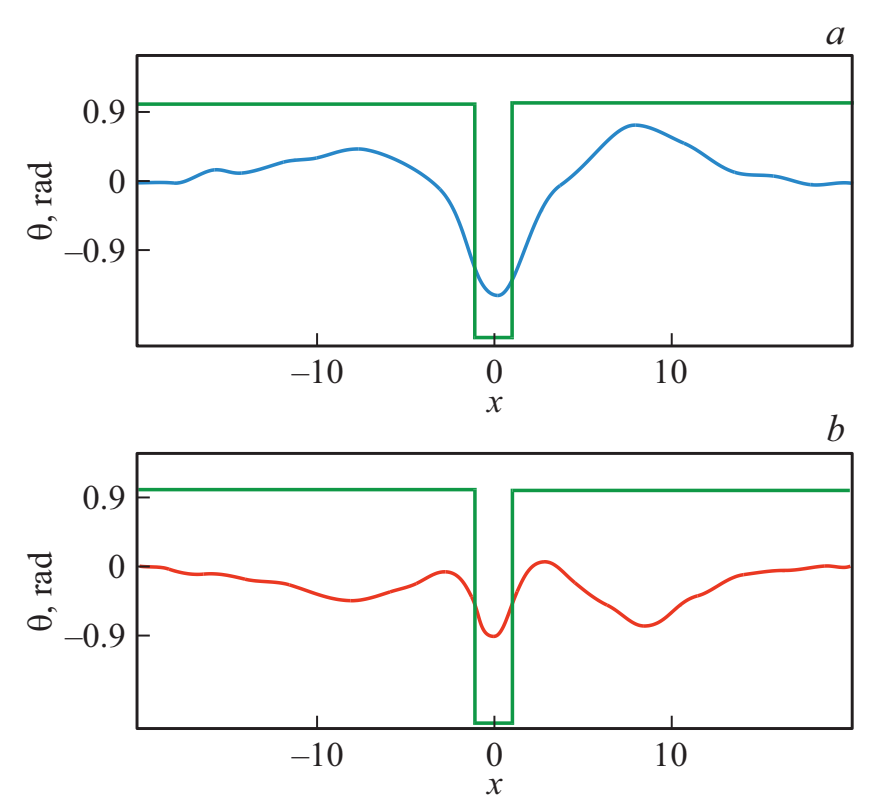

Рис. 3. Колебания антисолитона с излучением спиновых волн в отсутствие затухания в различные моменты времени. $t=153\left(\right.$ a) и $156(b) . W=2, K=-2, h_{0}=0.1, \mu=0.01$, начальная частота поля 1.0 .

Из проведенного исследования следует, что авторезонансная модель управления магнитным солитоном в трехслойном ферромагнетике позволяет пользоваться малыми по амплитуде переменными полями, что может найти применение в магнитных устройствах памяти.

\section{Финансирование работы}

Исследования Е.Г. Екомасова и К.Ю. Самсонова выполнены при финансовой поддержке Российского фонда фундаментальных исследований в рамках научного проекта № 20-31-90048, В.Н. Назарова — в рамках государственного задания \#АААА-А19-119022290052-9.

\section{Конфликт интересов}

Авторы заявляют, что у них нет конфликта интересов.

\section{Список литературы}

[1] C. Li, S. Wang, N. Xu, X. Yang, B. Liu, B. Yang, L. Fang, J. Magn. Magn. Mater., 498, 166155 (2019). DOI: $10.1016 /$ j.jmmm.2019.166155

[2] L. Shen, J. Xia, G. Zhao, X. Zhang, M. Ezawa, O.A. Tretiakov, X. Liu, Y. Zhou, Appl. Phys. Lett., 114, 042402 (2019). DOI: $10.1063 / 1.5080302$

[3] М.А. Шамсутдинов, И.Ю. Ломакина, В.Н. Назаров, А.Т. Харисов, Д.М. Шамсутдинов, Ферро- и антиферромагнитодинамика. Нелинейные колебания, волны и солитоны (Наука, М., 2009).

[4] R. Kukreja, S. Bonetti, Z. Chen, D. Backes, Y. Acremann, J.A. Katine, A.D. Kent, H.A. Dürr, H. Ohldag, J. Stöhr, Phys. Rev. Lett., 115, 096601 (2015).

DOI: 10.1103/PhysRevLett.115.096601

[5] M.V. Gerasimov, M.V. Logunov, A.V. Spirin, Yu.N. Nozdrin, I.D. Tokman, Phys. Rev. B, 94, 014434 (2016).

DOI: 10.1103/PhysRevB.94.014434 
[6] D. Backes, F. Macià, S. Bonetti, R. Kukreja, H. Ohldag, A.D. Kent, Phys. Rev. Lett., 115, 127205 (2015). DOI: 10.1103/PhysRevLett.115.127205

[7] P.J. Metaxas, M. Albert, S. Lequeux, V. Cros, J. Grollier, P. Bortolotti, A. Anane, H. Fangohr, Phys. Rev. B, 93, 054414 (2016). DOI: 10.1103/PhysRevB.93.054414

[8] E.G. Ekomasov, A.M. Gumerov, R.R. Murtazin, R.V. Kudryavtsev, A.E. Ekomasov, N.N. Abakumova, Solid State Phenom., 233-234, 51 (2015).

DOI: 10.4028/www.scientific.net/SSP.233-234.51

[9] E.G. Ekomasov, R.R. Murtazin, V.N. Nazarov, J. Magn. Magn. Mater., 385, 217 (2015). DOI: 10.1016/j.jmmm.2015.03.019

[10] А.М. Гумеров, Е.Г. Екомасов, Р.В. Кудрявцев, М.И. Фахретдинов, Письма о материалах, 8 (3), 299 (2018). DOI: 10.22226/2410-3535-2018-3-299-304

[11] E.G. Ekomasov, R.R. Murtazin, O.B. Bogomazova, A.M. Gumerov, J. Magn. Magn. Mater., 339, 133 (2013). DOI: 10.1016/j.jmmm.2013.02.042

[12] D.D. Tang, Y.-J. Lee, Magnetic memory: fundamentals and technology (Cambridge University Press, N.Y., 2010). DOI: $10.1017 /$ CBO9780511676208

[13] V.N. Nazarov, L.A. Kalyakin, M.A. Shamsutdinov, Solid State Phenom., 81, 168 (2011). DOI: $10.4028 /$ www.scientific.net/SSP.168-169.81

[14] Е.Г. Екомасов, В.Н. Назаров, А.М. Гумеров, К.Ю. Самсонов, Р.Р. Муртазин, Письма о материалах, 10 (2), 141 (2020). DOI: $10.22226 / 2410-3535-2020-2-141-146$

[15] Л.А. Калякин, М.А. Шамсутдинов, ТМФ, 160 (1), 102 (2009). [Пер. версия: 10.1007/s11232-009-0086-3].

[16] С.В. Баталов, А.Г. Шагалов, ФММ, 109 (1), 3 (2010). [Пер. версия: 10.1134/S0031918Х10010011].

[17] С.В. Баталов, А.Г. Шагалов, ФММ, 114 (2), 115 (2013). [Пер. версия: 10.1134/S0031918X13020038]. 